\title{
WISSENSCHAFTLICHE PAPERBACKS
}

\section{Sozial- und Wirtschaftsgeschichte}

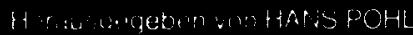

Je Band ca. 200 Seiten, DM 18,-, bei Reihenbezug DM 16,-

\section{Römische Sozialgeschichte}

\author{
Von Géza Alföldy 1975. Ca. 200 Seiten ISBN 3-515-02045-4
}

In der Arbeit wird erstmals versucht, die römische Sozialgeschichte von den Anfängen Roms bis zum Zerfall des Imperium Romanum in der Form einer populären, zugieich wissenschaftlich fundierten Darstellung zusammenzufassen. Den Gegenstand des Werkes bilden die wirtschaftlichen Grundlagen für die Sozialordnung, das Schichtungssystem, die einzelnen sozialen Schichten, die sozialen Abhängigkeitsformen, die sozialen Spannungen und Konflikte, die soziale Mobilität und der politisch-ideologische Rahmen für die Sozialordnung in den einzelnen Epochen der römischen Geschichte.

Bereits lieferbar:

I Ernst Klein: Geschichte der deutschen Landwirtschaft im Industriezeitalter 1973. VIII, 192 Seiten. ISBN 3-515-01190-0

2 Hans Jaeger: Geschichte der amerikanischen Wirtschaft im 20. Jahrhundert 1973. VIII, 186 Seiten. ISBN 3-515-01188-9

3 Fritz Blaich: Die Epoche des Merkantilismus 1974. VIII, 203 Seiten. ISBN 3-515-01197-8

4 Harald Winkel: Die Wirtschaft im geteilten Deutschland 1945-r970 1974. XIV, 217 Seiten. ISBN 3-515-01799-2

5 Albin Gladen: Geschichte der Sozialpolitik in Deutschland Eine Analyse ihrer Bedingungen, Formen, Zielsetzungen und Auswirkungen 1974. X, 201 Seiten. ISBN 3-515-01807-7

6 Ernst Klein: Geschichte der öffentlichen Finanzen in Deutschland (1500-1870)

1974. VIII, 145 Seiten. ISBN 3-515-01880-8

7 Winfried Baumgart: Der Imperialismus

Idee und Wirklichkeit der englischen und französischen Kolonialexpansion 1880-1914

1975. VIII, 179 Seiten, 1 Karte. ISBN 3-515-01969-3

In Vorbereitung:

Thomas Pekáry: Die Wirtschaft der griechischen und römischen Antike

FRANZ STEINER VERLAG GMBH • WIESBADEN 


\section{A N N A L E S \\ ECONOMIES - SOCIÉTÉS - CIVILISATIONS}

Revue bimestrielle, fondée en 1929 par

LUCIEN FEBVRE et MARC BLOCH

Comité de Divection:

FERNAND BRAUDEL, MARC FERRO, GEORGES FRIEDMANN, JACQUES LE GOFF, EMMANUEL LE ROY LADURIE, CHARLES MORAZÉ

Secrétaires $d u$ Comité: PAUL LEUILLIOT, MARIANNE MAHN-LOT, ANDRE BURGUIÈ RE Secretaire de Rédaction: JACQUES REVEL

30e ANNÉE - No 4 - JUILLET-AOOT 1975

\section{Echanges culturels}

W. G. L. Randles, Echanges de marchandises et échanges de dieux

David Nicholls, Idéologie et mouvements politiques en Haiti, 1915-1946

\section{Démographie et société}

Michel Terrisse, A propos de démographie médiévale: de moderniste à médiéviste, notes de lecture

John Day, Malthus démenti? Souspeuplement chronique et catastrophes démographiques en Sardaigne au Bas Moyen Age

Gérard Delille, Types de développement dans le royaume de Naples, XVIIeXVIIIe siècle

Emmanuel Todd, Mobilité géographique et cycle de vie en Artois et en Toscane au XVIIIe siècle

\section{L'Espace parisien}

Guy Arbellot, Les barrières de l'An VII

Françoise Boudon, Tissu urbain et architecture. L'analyse parcellaire comme base de l'histoire architecturale

Gérard Jacquemet, Belleville aux XIXe et XXe siècles: une méthode d'analyse de la croissance urbaine à Paris

Comptes rendus: A travers la France

\section{La civilisation islamique}

Gilles Hennequin, De la monnaie antique à la monnaie musulmane Hichem Djait, L'Islam ancien récupéré à l'histoire Comptes rendus: Islam et monde arabe

Rédaction: 54, Boulevard Raspail, 75006-Paris

Administration: Librairie Armand Colin, 103, Boulevard Saint-Michel, 75005-Paris

Comptes chèques postaux: Paris, No 21 335-25

Abonnements: France et Pays de la Communauté: $100 \mathrm{~F}$ (Etudiants France: $70 \mathrm{~F}$ ) - Etranger : $130 \mathrm{~F}$ - Le numéro: $20 \mathrm{~F}$ - Numéros spéciaux: $40 \mathrm{~F}$ 